\title{
Templated J-Aggregate Nanotubes for the Detection of Dopamine
}

\author{
Samuel Rhodes', Xiaochen Wang1, Wenlang Liang1, Hyoung Jin Cho², Jiyu Fang1* \\ ${ }^{1}$ Department of Materials Science and Engineering, University of Central Florida, Orlando, USA \\ ${ }^{2}$ Department of Mechanical and Aerospace Engineering, University of Central Florida, Orlando, USA \\ Email: *jiyu.fang@ucf.edu
}

How to cite this paper: Rhodes, S., Wang, X.C., Liang, W.L., Cho, H.J. and Fang, J.Y. (2017) Templated J-Aggregate Nanotubes for the Detection of Dopamine. Journal of Materials Science and Chemical Engineering, 5, 142-154.

http://dx.doi.org/10.4236/msce.2017.51018

Received: November 30, 2016

Accepted: January 20, 2017

Published: January 23, 2017

Copyright $\odot 2017$ by authors and Scientific Research Publishing Inc. This work is licensed under the Creative Commons Attribution International License (CC BY 4.0).

http://creativecommons.org/licenses/by/4.0/ (c) $\underset{\mathrm{EY}}{\text { (i) Open Access }}$

\begin{abstract}
J-aggregates of dye molecules are a unique supramolecular structure, which shows great promise in photoelectric devices due to its remarkable optical and transport properties. In this paper, we report the templated formation of J-aggregate nanotubes by the adsorption of 3,3'-diethylthiacarbocyanine iodide on the self-assembled nanotubes of lithocholic acid. The optical and electronic properties of the templated J-aggregate nanotubes are studied. A sensor platform is fabricated by depositing the J-aggregate nanotubes on interdigitated gold electrodes for the detection of dopamine (DA). We find that the current change of the J-aggregate nanotube-based sensor platform in response to DA is linear in the concentration range from $10 \mathrm{nM}$ to $70 \mathrm{nM}$, giving the detection limit of $0.27 \mathrm{nM}$.
\end{abstract}

\section{Keywords}

Cyanine Dyes, Bile Acids, J-Aggregate Nanotubes, Dopamine, Detection

\section{Introduction}

J-aggregates of dye molecules represent an organized supramolecular structure, in which molecules form a head-to-tail staircase arrangement [1]. The strong interaction of transition dipole moments in J-aggregates leads to new electronic excitations. The new electronic excitations in J-aggregates are coherently delocalized over many molecules rather than confined to individual molecules. The delocalized excitation of J-aggregates leads to an intense absorption band (J-band) and favors for efficient electron transfer. Therefore, they have long been used for mimicking photosynthetic light-harvesting antennas [2] [3] [4] [5]. Due to the remarkable optical and transport properties, J-aggregates have also shown great promise in color photography [6], nonlinear optics [7] [8], light emitting diodes [9] [10], and solar cells [11] [12]. However, the application of J-aggregates in 
biosensors is still limited [13] [14] [15]. This is largely due to the poor stability of $\mathrm{J}$-aggregates in biological fluids, in which the interaction of J-aggregates with proteins and salts may change their structures and consequently optical properties. Recently, efforts have been made in stabilizing J-aggregates by assembling them on lipid vesicles [14], lipid nanotubes [15], DNA helix [16], $\beta$-sheet fibrils [17], and peptide nanotubes [18].

J-aggregate nanotubes are attractive for efficient electron transfers because they share the molecular packing feature with photosynthetic light-harvesting antennas [19]-[24]. In this paper, we report the formation of templated J-aggregate nanotubes by the adsorption of 3,3'-diethylthiacarbocyanine iodide (DTCC) on the self-assembled nanotubes of lithocholic acid (LCA). The optical and electronic properties of the templated J-aggregate nanotubes are investigated with UV-vis absorption spectra, cyclic voltammetry methods and currentvoltage measurements. We fabricate a sensor platform by depositing the templated J-aggregate nanotubes on interdigitated gold electrodes for the detection of dopamine (DA). DA is known to be an important neurotransmitter in central and peripheral nervous systems [25]. The concentration level of DA is associated with a series of neural diseases including Parkinson's and Alzheimer's diseases [26]. It is for this reason that DA has been long used as an important biomarker in the diagnosis of these neural diseases. Electrochemical methods [27] [28], capillary electrophoresis [29] [30], and liquid chromatography-mass spectrometry [31] are conventional analytical methods for the detection of DA. However, these methods are time consuming and require expensive instruments. The sensitivity of the J-aggregate nanotube-based sensor platform for DA is studied by measuring the photo-induced current change as a function of DA concentrations. We find that the detection limit of the J-aggregate nanotube-based sensor platform for DA is $0.27 \mathrm{nM}$, which is two orders of magnitude lower than these conventional analytical methods.

\section{Experimental Section}

\subsection{Materials}

Lithocholic acid (LCA), $\mathrm{NH}_{4} \mathrm{OH}$ solution, dopamine (DA), and uric acid (UA) were purchased from Sigma-Aldrich. 3,3'-diethylthiacarbocyanine iodide (DTCC) was purchased from Molecular Probes. All chemicals were used without further purification. Water used in the experiments was purified with Easypure II system (18 $\mathrm{M} \Omega \mathrm{cm}, \mathrm{pH}$ 5.7). Silicon wafer and carbon coated copper grids were from Sigma-Aldrich and Electron Microscopy Science, respectively.

\subsection{Formation of Templated J-Aggregate Nanotubes}

LCA was added into $10 \% \mathrm{w} / \mathrm{w} \mathrm{NH}_{4} \mathrm{OH}$ solutionin a glass vial to achieve a final concentration of $3 \mathrm{mM}$, followed by $5 \mathrm{~min}$ sonication in an ultrasonic bath (Branson 1510, Branson Ultrasonics Co.) at $50^{\circ} \mathrm{C}$. LCA nanotubes were formed when LCA solution was cooled to room temperature. Once LCA nanotubes were formed, $0.13 \mathrm{Mm}$ DTCC was added. The mixture was incubated overnight for 
the adsorption of DTCC on LCA nanotubes, leading to the formation of templated J-aggregate nanotubes, which were then purified through centrifugation to remove excess DTCC. The templated J-aggregate nanotubes are stable for weeks in dark. There is no adsorption spectrum change observed.

\subsection{Characterization}

The morphology of LCA nanotubes and templated J-aggregate nanotubes was studied with a transmission electron microscope (TEM, FEW Technai F30), a scanning electron microscope (SEM, Hitachi S3500N), and an atomic force microscope (AFM, Dimension 3100 Veeco Instruments). For the TEM measurements, nanotubes were dried on carbon coated copper grids. AFM images of nanotubes dried on glass substrates were taken with a silicon nitride cantilever (Nanosensors) with a spring constant of $30 \mathrm{~N} / \mathrm{m}$ and a resonant frequency of 260 $\mathrm{kHz}$ in tapping mode. The optical properties of J-aggregate nanotubes were characterized with a Cary 300 UV-Vis spectrophotometer. Cyclic voltammetry (CV) measurements were performed in aqueous solution containing $1 \mathrm{M} \mathrm{KCl}$ using a CS 350 Electrochemical workstation, in which templated J-aggregate nanotubes were deposited on an ITO working electrode, a platinum wire acted as the counter electrode and $\mathrm{Ag} / \mathrm{AgCl}$ served as a reference electrode. Current-voltage (I-V) measurements were performed with a Keithley 2400 sourcemeter.

\subsection{Fabrication of Interdigitated Gold Electrodes}

For the fabrication of interdigitated gold $(\mathrm{Au})$ electrodes, a $100 \mathrm{~nm}$ thick $\mathrm{SiO}_{2}$ insulation layer was first grown on a 3-inch $\mathrm{Si}$ wafer by thermal oxidation process. A metallic seed film which consists of $100 \mathrm{~nm}$ Au layer over $10 \mathrm{~nm} \mathrm{Ti}$ layer was then deposited on the top of the $\mathrm{SiO}_{2}$ layer by e-beam evaporation. A thin layer of positive photoresist (Microposit S1813, Dow Electronic Materials) was then spin-coated $(3000 \mathrm{rpm}, 30 \mathrm{sec})$, soft-baked $\left(105^{\circ} \mathrm{C}, 3 \mathrm{~min}\right)$, exposed under I-line UV aligner (8 seconds) and developed (Microposit MF CID-26 Developer, $35 \mathrm{sec}$ ) to obtain photoresist patterns for the interdigitated electrodes with a gap of $20 \mu \mathrm{m}$. The developed patterns were hard-baked at $105^{\circ} \mathrm{C}$ for 10 $\mathrm{min}$ ) and used as a protective layer for subsequent etching steps. The Au/Ti seed layer in exposed areas was chemically etched with an $\mathrm{Au}$ etchant (KI and $\mathrm{I}_{2}$ mixture solution) and a Ti etchant $(0.5 \% \mathrm{HF})$. The remaining photoresist was dissolved in acetone and the interdigitated $\mathrm{Au}$ electrodes were formed after cleaning in methanol and deionized water. The schematic illustration of the fabrication of the interdigital gold electrodes is shown in Figure 1.

\subsection{Fabrication of J-Aggregate Nanotube-Based Sensor Platform}

The interdigitated $\mathrm{Au}$ electrodes were cleaned with deionized water before the deposition of templated J-aggregate nanotubes. In our experiments, $4 \mu \mathrm{L}$ J-aggregate nanotube solution was dropped on the interdigitated Au electrodes and dried in air at room temperature. The J-aggregate nanotube-based sensor platform was immersed in buffer solution at $\mathrm{pH} 10$ for 1 hour, followed by the 
addition of DA in the dark. After 30 min incubation, the J-aggregate nanotubebased sensor was irradiated with visible light for 30 seconds, followed by measuring current changes with a sourcemeter.

\section{Results and Discussion}

The chemical structures of DTCC and LCA are shown in Figure 2. DTCC is a positively charged carbocyanine dye consisting of two nitrogen-containing heterocyclic ring systems, which are linked by a methane bridge. LCA is a secondary

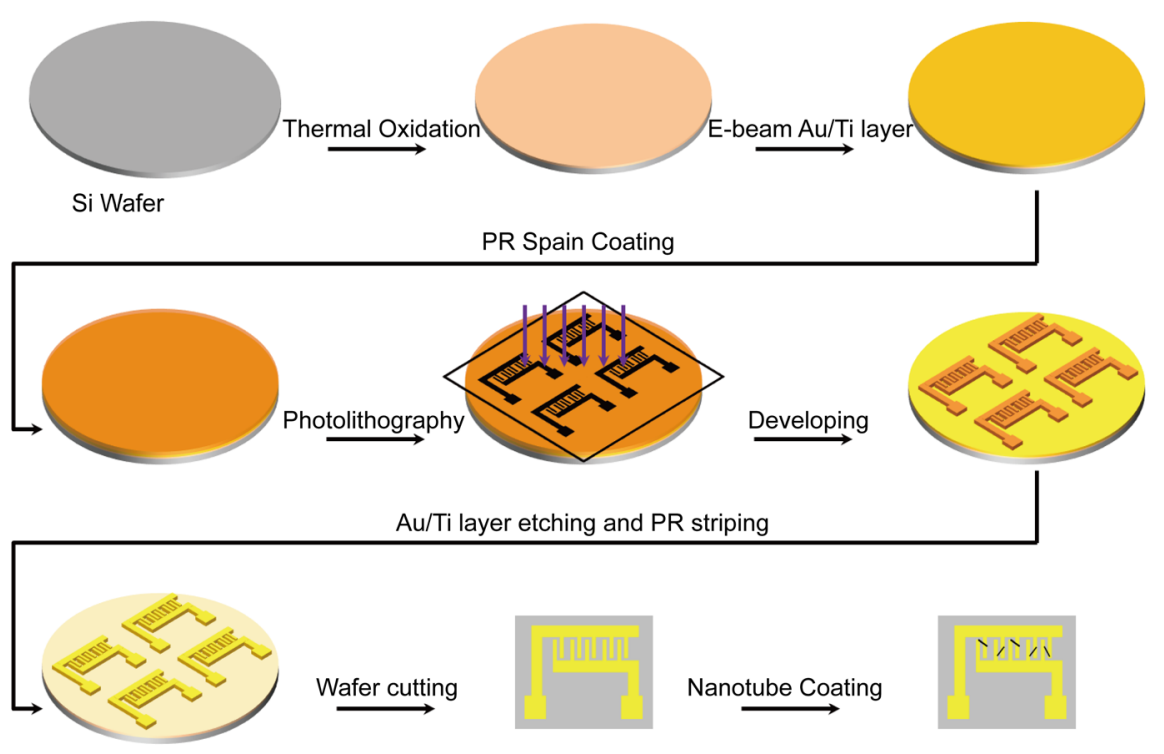

Figure 1. Schematic illustration of the fabrication of interdigital gold electrodes.

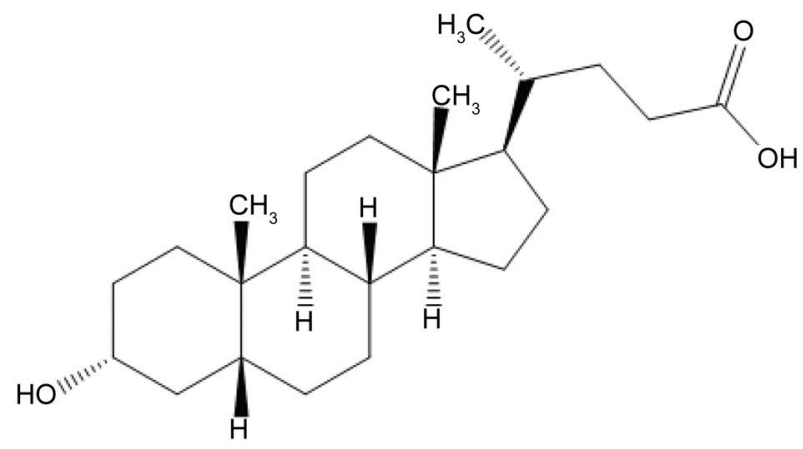

(a)<smiles>CCN1/C(=C/C=C/c2sc3ccccc3[n+]2C)Sc2ccccc21</smiles>

(b)

Figure 2. Chemical structure of lithocholic acid (a) and 3, 3'-diethylthiacarbocyanine iodide (b). 
bile acid having a nearly planar steroid backbone and a carboxyl group linked to the steroid backbone through a short alkyl chain. It has been shown that negatively charged LCA in alkaline aqueous solution can self-assemble into tubular structures with different diameters and shapes, depending on experimental conditions [32]-[37]. Figure 3(a) is an AFM image of LCA nanotubes formed in $\mathrm{NH}_{4} \mathrm{OH}$ solution. The LCA nanotubes show a smooth surface and a uniform diameter along their long axis. The hollowness of the LCA nanotubes is clear visible in the TEM image shown in Figure 3(b). They have an external diameter of $\sim 80 \mathrm{~nm}$ and a wall thickness of $\sim 15 \mathrm{~nm}$. The wall thickness of the LCA nanotubes is larger than the length of the LCA molecule $(\sim 1.6 \mathrm{~nm})$, suggesting that the wall of the LCA nanotubes has a multi-lamellar structure.

After the adsorption of positively charged DTCC through electrostatic interaction, the surface of negatively charged LCA nanotubes becomes rough (Figure 4(a)).
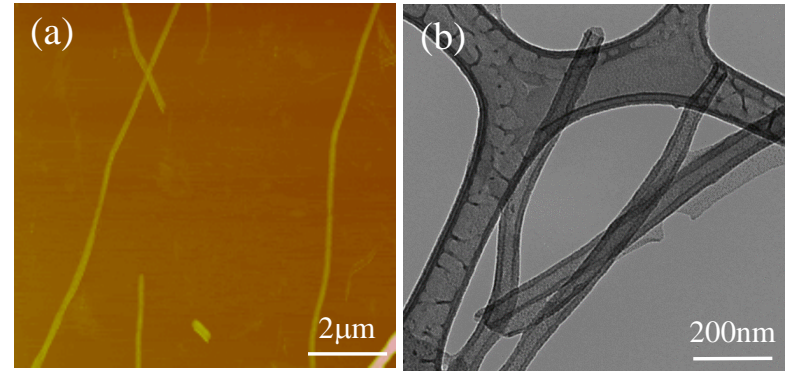

Figure 3. (a) AFM of LCA nanotubes dried on glass substrates and (b) TEM images of LCA nanotubes dried on a carbon coated copper grid.
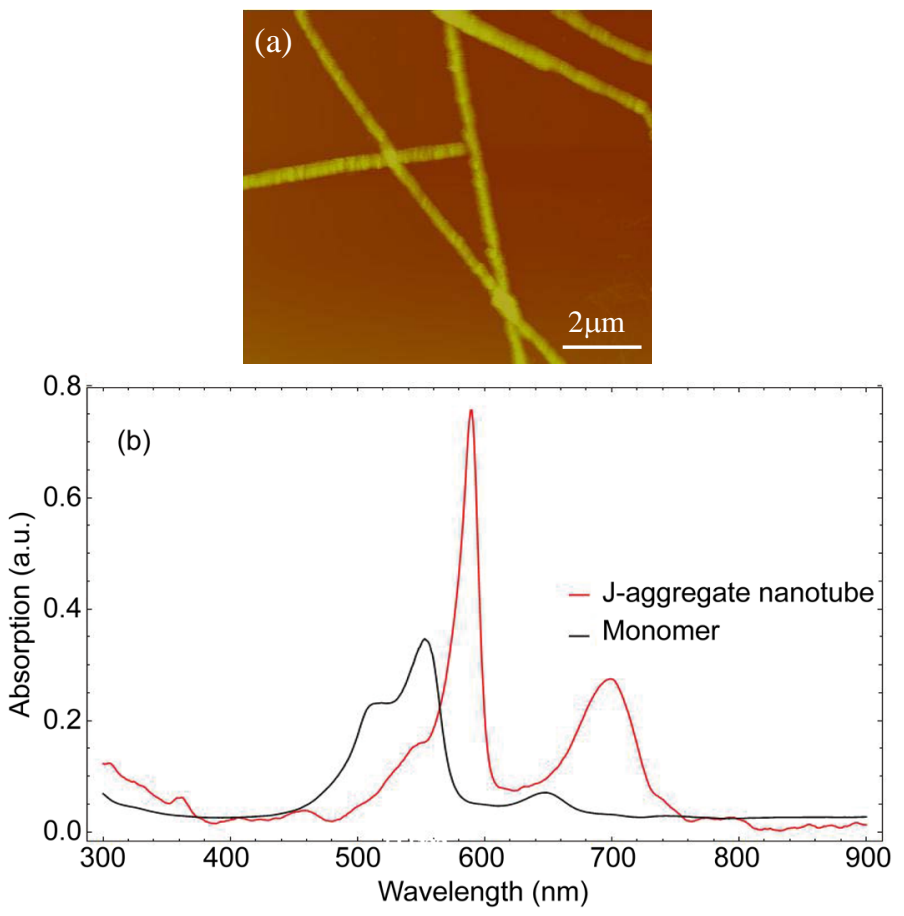

Figure 4. (a) AFM image of templated J-aggregate nanotubes dried on a glass sub-strate. (b) Absorption spectra of templated J-aggregate nanotubes and DTCC mono-mer in $\mathrm{NH}_{4} \mathrm{OH}$ solution. 
UV-vis absorption spectra show that the DTCC molecules adsorbed on LCA nanotubes form J-aggregates (Figure 4(b)). The templated J-aggregate nanotubes show an intense adsorption band (J-band) at $590 \mathrm{~nm}$, which is red-shifted with respect to the adsorption band of DTCC monomer at $550 \mathrm{~nm}$. The delocalized excitation length of J-aggregates, which represents the number of coherently coupled molecules $\left(N_{\text {coh }}\right)$ in the J-aggregates, can be calculated from their absorption spectra according to the equation [38] [39]:

$$
N_{\text {coh }}=\frac{3\left(\Delta v_{\text {mon }}\right)^{2}}{2\left(\Delta v_{J}\right)^{2}}-1
$$

where $\Delta v_{\text {mon }}$ and $\Delta v_{J}$ are the full widths at the half maxima of monomer and J-aggregate absorption bands, respectively. Thus, we use the equation 1 to calculate the delocalized excitation length of the templated J-aggregate nanotubes from their absorption spectra to be 22 monomers.

The templated J-aggregate nanotubes were deposited on the interdigitated $\mathrm{Au}$ electrodes with a gap of $20 \mu \mathrm{m}$ for measuring their electronic properties (Figure 5(a)). As can be seen in Figure 5(b), the templated J-aggregate nanotubes form a network bridging the interdigitated Au electrodes. Individual J-aggregate nanotubes without metallic coating are visible in the SEM image shown in Figure 5(c). The formation of J-aggregate nanotube networks on interdigitated Au electrodes provides a large number of conductive paths for the connection of external circuits.

Figure 6 shows the current-voltage (I-V) curves of J-aggregate nanotube networks in the applied voltage from $-5 \mathrm{~V}$ to $+5 \mathrm{~V}$. These I-V curves are symmetrical. There is no change in the I-V curve observed when the J-aggregate nanotube network is exposed to visible light irradiation (Figure 6). We note that the $\mathrm{J}$-aggregate nanotube networks deposited on the interdigitated $\mathrm{Au}$ electrodes with the gap of $20 \mu \mathrm{M}$ are stable in buffer solution over time and during multiple measurements. However, when the gap of interdigitated Au electrodes reduces to $8 \mu \mathrm{M}$, the deposited J-aggregate nanotube networks become unstable after being immersed in buffer solution. This result suggests that the contact of J-aggregate nanotubes with the $\mathrm{SiO}_{2}$ layer plays an important role for immobilizing them on the interdigitated Au electrodes.

We exploit the application of the J-aggregate nanotube network deposited on the interdigitated $\mathrm{Au}$ electrodes with the gap of $20 \mu \mathrm{M}$ as a sensor platform for
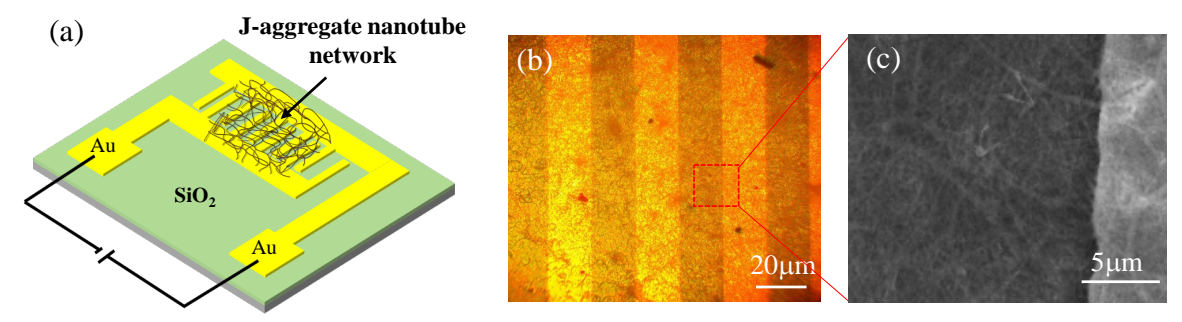

Figure 5. (a) Schematic of J-aggregate nanotube-based sensor platform. (b) Optical microscopy (a) and SEM (b) images of interdigitated Au electrodes with deposited J-aggregate nanotube networks. 


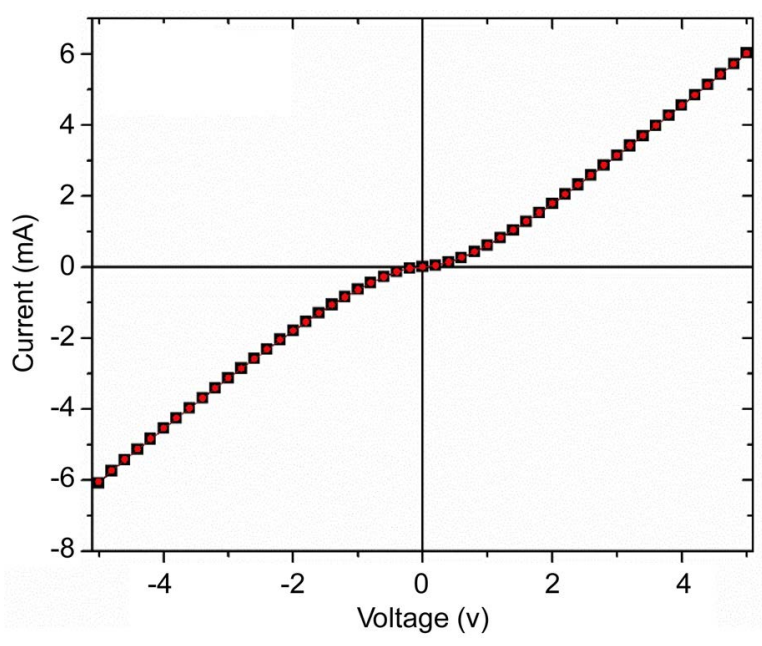

Figure 6. I-V curves of J-aggregate nanotube-based sensors with $(\bullet)$ and without $(\square)$ visible light irradiation.

the detection of DA in alkaline buffer solution. It has been shown that DA has $\mathrm{pH}$-dependent oxidation and reduction potentials (Figure 7) [40]. In alkaline buffer solution, DA is easily oxidized into quinone by oxygen. DA-quinone is an electron acceptor [41]. In our experiments, the J-aggregate nanotube based sensor platform was immersed in $50 \mathrm{~mL}$ buffer solution at $\mathrm{pH} \mathrm{10,} \mathrm{followed} \mathrm{by} \mathrm{the}$ addition of DA in the dark. It has been shown that DA is able to adsorb on a variety of organic surfaces through strong intermolecular interactions including hydrogen-bonding, $\pi-\pi$ interaction, and metal chelation [42]. The J-aggregate nanotubes are stable in the buffer solution at $\mathrm{pH} 10$. There is no adsorption spectrum change observed. After $30 \mathrm{~min}$ incubation at room temperature for the oxidization and adsorption of DA on the J-aggregate nanotube networks, the J-aggregate nanotube-based sensor was irradiated with visible light for 30 seconds, followed by measuring the current change under the forward voltage at $5 \mathrm{~V}$. To avoid sensor-to-sensor variations, we used the normalized current change $\Delta \mathrm{I} / \mathrm{I}_{0}$ to represent the current response of J-aggregate nanotube-based sensors to DA, where $\Delta \mathrm{I}$ is $\mathrm{I}_{0}-\mathrm{I}, \mathrm{I}_{0}$ is the current without $\mathrm{DA}$, and $\mathrm{I}$ is the current with DA. As can be seen in Figure 8, the normalized current change of J-aggregate nanotube based sensors after the addition of $20 \mathrm{nM} \mathrm{DA}$ at the forward voltage of $5 \mathrm{~V}$ with visible light irradiation is much higher than that without light irradiation. Thus, we infer that the current change of the J-aggregate nanotube-based sensor in response to DA under visible light irradiation is a result of the photoinduced electron transfer (PET) from the excited J-aggregate nanotubes to the adsorbed DA-quinone (see the schematic insert in Figure 8).

It is known that the relative HOMO and the LUMO levels between a donor and an acceptor determines the electron transfer. Cyclic voltammetry (CV) measurements show the irreversible oxidation of the templated J-aggregate nanotubes immobilized on ITO in aqueous solution containing $1 \mathrm{M} \mathrm{KNO}_{3}$ (Figure 9). The templated J-aggregate nanotubes are oxidized at the positive potential of $+0.43 \mathrm{~V}$ with no corresponding reduction peak. The highest occupied molecular 

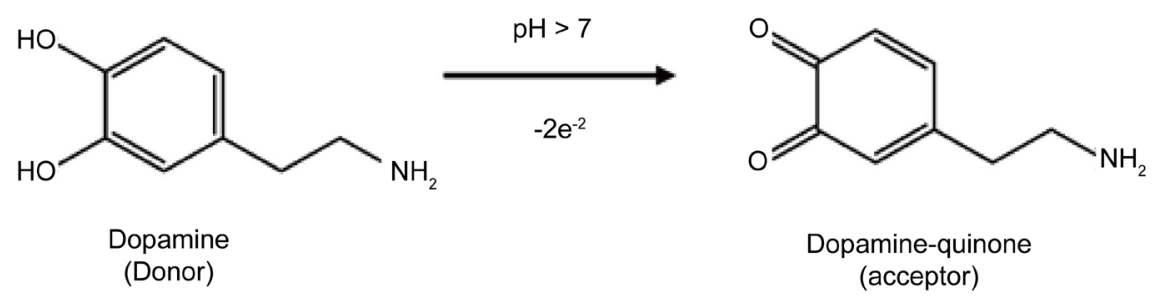

Figure 7. Schematic illustration of the oxidization of DA induced by $\mathrm{pH}$ values.

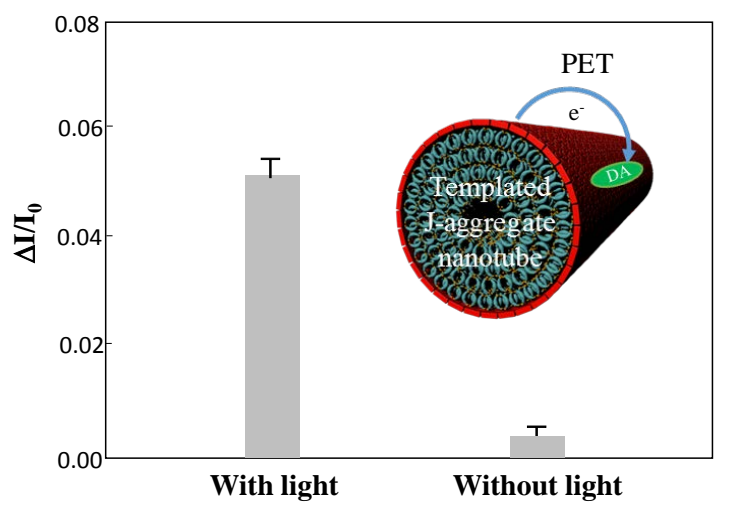

Figure 8. Current change of J-aggregate nanotube-based sensor platform in response to $20 \mathrm{nM} \mathrm{DA}$ in buffer solution at $\mathrm{pH} 10$ with and without 30 $s$ visible light irradiation. Schematic of photoinduced electron transfer (PET) from the templated J-aggregate nanotube to the adsorbed DA is insert in Figure 8.

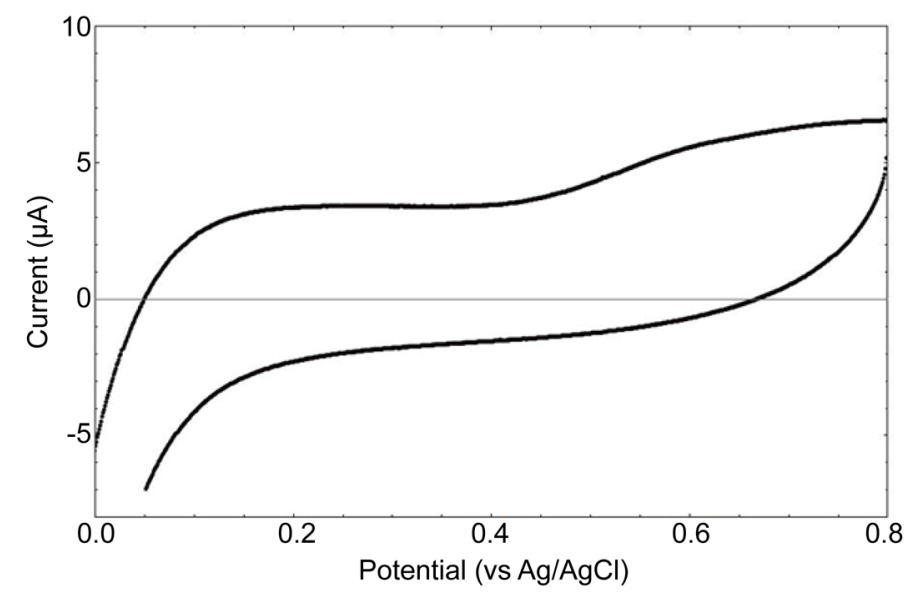

Figure 9. Cyclic voltammogram of J-aggregate nanotubes immobilized on ITO in aqueous solution containing $1 \mathrm{M} \mathrm{KCl}$. Scan rate: $0.1 \mathrm{~V} / \mathrm{s}$.

orbital (HOMO) and the lowest unoccupied molecular orbital (LUMO) levels of $J$-aggregate nanotubes can be calculated with the following empirical equations [43]:

$$
\begin{aligned}
& E(\mathrm{HOMO})=-e\left(E_{\text {onset }}+4.4\right) \\
& E(\mathrm{LUMO})=E_{g}-E(\mathrm{HOMO})
\end{aligned}
$$

where $E_{\text {onset }}$ is $4.3 \mathrm{~V}$ and $E_{g}$ is the optical band gap energy which can be calculated according to the equation [44]: $E_{g}=1242 / \lambda_{\text {onset. }}$. For the templated J-aggregate 
nanotubes, $\lambda_{\text {onset }}$ is $590 \mathrm{~nm}$. Thus, $E_{g}$ is calculated to be $2.10 \mathrm{eV}$. Using the empirical equations, we find that the $E(\mathrm{HOMO})$ and $E(\mathrm{LUMO})$ level of the templated J-aggregate nanotubes is $-4.83 \mathrm{eV}$ and $-2.73 \mathrm{eV}$, respectively. The LUMO level of DA-quinone is $-3.85 \mathrm{eV}$ [45], which is inside the the HOMO-LUMO energy gap of the templated J-aggregate nanotubes, favoring the electron transfer from the excited J-aggregate nanotube to the adsorbed DA-quinone.

The current change due to the PET from the excited J-aggregate nanotubes to the adsorbed DA-quinone provides a basis for the detection of DA. Figure 10(a) shows a plot of $\Delta \mathrm{I} / \mathrm{I}_{0}$ as a function of DA concentrations in buffer solution at $\mathrm{pH}$ 10. The plot can be fitted linearly with a correlation coefficient of 0.9748 in the concentration range from $10 \mathrm{nM}$ to $70 \mathrm{nM}$, which falls within the concentration level of DA for patients with Parkinson's disease [46]. The detection limit of J-aggregate nanotube sensors for DA is calculated by multiplying the ratio of the standard deviation to the slope of the linear fit curve by 3.3 to be $\sim 0.27 \mathrm{nM}$. The detection of DA is often complicated by the presence of uric acid (UA), which coexists with DA in vivo at a concentration of 100 - 1000 times higher than DA [47]. We find that the current response of the J-aggregate nanotube-based sensors for DA is significantly higher than that for UA even when the concentration of UA is 1000 times higher than DA (Figure 10(b)). The selectivity of J-aggregate nanotube-based sensors for DA should associate with its high affinity to the J-aggregate nanotubes and the efficient electron transfer between them.
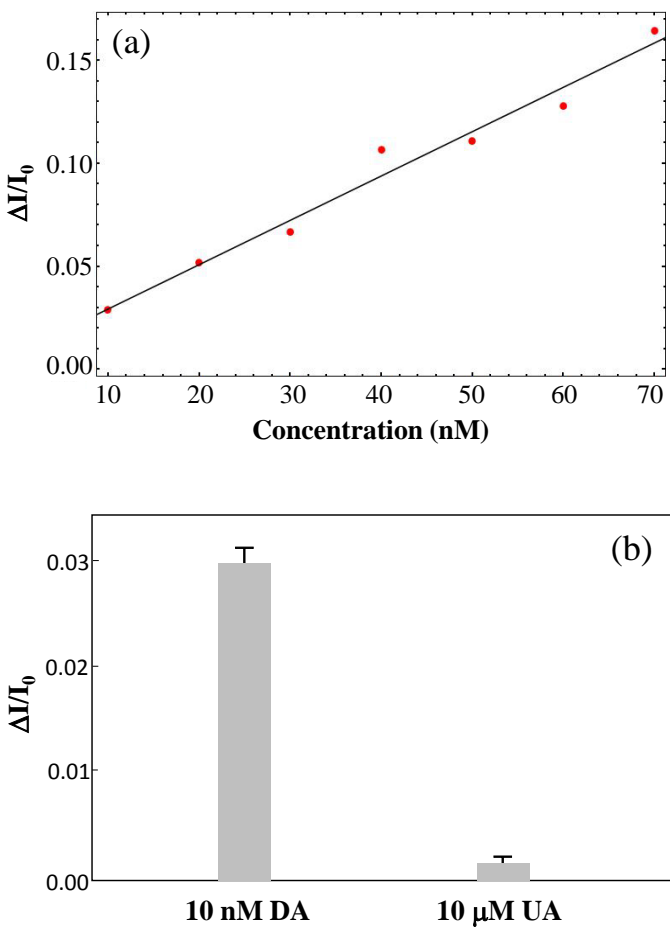

Figure 10. (a) Current changes of J-aggregate nanotube-based sensor platform as a function of DA concentrations in buffer solution at $\mathrm{pH} 10$ with $30 \mathrm{~s}$ visible light irradiation. (b) Current changes of J-aggregate nanotube-based sensor platform in response to $10 \mathrm{nM}$ DA and $10 \mu \mathrm{M}$ UA in buffer solution at $\mathrm{pH} 10$ with $30 \mathrm{~s}$ visible light irradiation. 


\section{Conclusion}

We have shown that the adsorption of DTCC on self-assembled LCA nanotubes can lead to the formation of J-aggregate nanotubes. The templated J-aggregate nanotubes show an intense J-band at $590 \mathrm{~nm}$, which is red-shifted with respect to the adsorption band of DTCC monomer at $550 \mathrm{~nm}$. A sensor platform is fabricated by forming the network of the templated J-aggregate nanotubes on interdigitated $\mathrm{Au}$ electrodes for the sensitive and selective detection of DA. The J-aggregate nanotube networks provide a high density of J-aggregate nanotubes for the accessibility of DA and a large number of conductive paths to external circuits. The photo-induced electron transfer from the J-aggregate nanotubes to the adsorbed DA leads to the current change of the J-aggregate nanotube-based sensor, which serves as the basis for the detection of DA. We find that the current change of the J-aggregate nanotube-based sensor in response to DA is linear in the concentration range from $10 \mathrm{nM}$ to $70 \mathrm{nM}$ with the detection limit of 0.27 $\mathrm{nM}$, which is significantly lower than that of conventional analytical methods reported in the literature. The high sensitivity is a result of efficient photoinduced electron transfer from the J-aggregate nanotubes to DA. The J-aggregate nanotube-based sensors are potable and open up an important avenue for the label-free and sensitive detection of DA.

\section{Acknowledgements}

This work was supported by US National Science Foundation (CBET-1264355).

\section{References}

[1] Jelley, E.E. (1936) Molecular, Nematic and Crystal States of I: I-Diethyl-Cyanine Chloride. Nature, 138, 2.

[2] Balaban, T.S. (2005) Tailoring Porphyrins and Chlorins for Self-Assembly in Biomimetic Artificial Antenna systems. Accounts of Chemical Research, 38, 612-623. https://doi.org/10.1021/ar040211z

[3] Scholes, G.D., Fleming, G.R., Olaya-Castro, A. and van Grondelle, R. (2011) Lessons from Nature about Solar Light Harvesting. Nature Chemistry, 3, 763-774.

https://doi.org/10.1038/nchem.1145

[4] Sengupta, S. and Würthner, F. (2013) Chlorophyll J-Aggregates: From Bioinspired dye Stacks to Nanotubes, Liquid Crystals, and Biosupramolecular Electronics. Accounts of Chemical Research, 46, 2498-2512. https://doi.org/10.1021/ar400017u

[5] Saikin, S.K., Eisfeld, A., Valleau, S. and Aspuru-Guzik, A. (2013) Photonics Meets Cxcitonics: Natural and Artificial Molecular Aggregates. Nanophotonics, 2, 21-38. https://doi.org/10.1515/nanoph-2012-0025

[6] Tani, T. (1996) J-Aggregates in Spectral Sensitization of Photographic Materials. In Kobayashi, T., Ed., J-Aggregates, World Scientific, Singapore, p. 209. https://doi.org/10.1142/97898128300290009

[7] Collini, E., Ferrante, C., Bozio, R., Lodi, A. and Ponterini, G. (2006) Large ThirdOrder Nonlinear Optical Response of Porphyrin J-Aggregates Oriented in SelfAssembled Thin Films. Journal of Materials Chemistry, 16, 1573-1578. https://doi.org/10.1039/b517591g 
[8] Cui, L., Tokarz, D., Cisek, R., Ng, K.K., Wang, F., Chen, J., Barzda, V. and Zheng, G. (2015) Organized Aggregation of Porphyrins in Lipid Bilayers for Third Harmonic Generation Microscopy. Angewandte Chemie International Edition, 54, 1392813932. https://doi.org/10.1002/anie.201506171

[9] Era, M., Adachi, C., Tsutsui, T. and Saito, S. (1991) Double-Heterostructure Electroluminescent Device with Cyanine-Dye Bimolecular Layer as An Emitter. Chemical Physics Letters, 178, 488-490. https://doi.org/10.1016/0009-2614(91)87007-X

[10] Maltsev, E.I., Lypenko, D.A., Shapiro, B.I., Brusentseva, M.A., Milburn, G.H.W., Wright, J., Hendriksen, A., Berendyaev, V.I., Kotov, B.V. and Vannikov, A.V. (1999) Electroluminescence of Polymer/J-Aggregate Composites. Applied Physical Letters, 75, 1896. https://doi.org/10.1063/1.124864

[11] Spencer, S., Cody, J., Misture, S., Cona, B., Heaphy, P., Rumbles, G., Andersen, J. and Collison, C. (2014) Critical Electron Transfer Rates for Exciton Dissociation Governed by Extent of Crystallinity in Small Molecule Organic Photovoltaics. Journal of Physical Chemistry C, 118, 14840-14847. https://doi.org/10.1021/jp504377r

[12] Chen, G., Sasabe, H., Lu, W., Wang, X.F., Kido, J., Hong, Z. and Yang, Y. (2013) J-Aggregation of a Squaraine Dye and Its Application in Organic Photovoltaic Cells. Journal of. Materials Chemistry A, 1, 6547-6552.

[13] Jones, R.M., Lu, L., Helgeson, R., Bergstedt, T.S., McBranch, D.W. and Whitten, D.G. (2001) Building Highly Sensitive Dye Assemblies for Biosensing from Molecular Building Blocks. Proceedings of the National Academy of Sciences USA, 98, 14769-14772. https://doi.org/10.1073/pnas.251555298

[14] Ng, K.K., Shakiba, M., Huynh, E., Weersink, R.A., Roxin, A., Wilson, B.C. and Zheng, G. (2014) Stimuli-Responsive Photoacoustic Nanoswitch for in Vivo Sensing Applications. ACS Nano, 8, 8363-8373. https://doi.org/10.1021/nn502858b

[15] Liang, W., He, S. and Fang, J.Y. (2014) Self-Assembly of J-Aggregate Nanotubes and Their Applications for Sensing Dopamine. Langmuir, 30, 805-811. https://doi.org/10.1021/la404022q

[16] Wang, W., Silva, G.L. and Armitage, B.A. (2000) DNA-Templated Formation of a Helical Cyanine Dye J-Aggregate. Journal of the American Chemical Society, 122, 9977-9986. https://doi.org/10.1021/ja002184n

[17] Berlepsch, H.V., Brandenburg, E., Koksch, B. and Bottcher, C. (2010) Peptide Adsorption to Cyanine Dye Aggregates Revealed by Cryo-Transmission Electron Microscopy. Langmuir, 26, 11452-11460. https://doi.org/10.1021/la100944d

[18] Kim, J.H., Lee, M., Lee, J.S. and Park, C.B. (2012) Self-Assembled Light-Harvesting Peptide Nanotubes for Mimicking Natural Photosynthesis. Angewandte Chemie International Edition, 51, 517-520. https://doi.org/10.1002/anie.201103244

[19] Von Berlepsch, H., Böttcher, C., Ouart, A., Burger, C., Dähne, S. and Kirstein, S. (2000) Supramolecular Structures of J-Aggregates of Carbocyanine Dyes in Solution. Journal of Physical Chemistry B, 104, 5255-5262. https://doi.org/10.1021/jp000220z

[20] Lang, E., Sorokin, A., Drechsler, M., Malyukin, Y.V. and Köhler, J. (2005) Optical Spectroscopy on Individual Amphi-PIC J-Aggregates. Nano Letters, 5, 2635-2640. https://doi.org/10.1021/nl051132z

[21] Kameta, N., Ishikawa, K., Masuda, M., Asakawa, M. and Shimizu, T. (2011) Soft Nanotubes Acting as a Light-Harvesting Antenna System. Chemistry of Materials, 24, 209-214. https://doi.org/10.1021/cm2030526

[22] Yuen-Zhou, J., Arias, D.H., Eisele, D.M., Steiner, C.P., Krich, J.J., Bawendi, M.G., Nelson, K.A. and Aspuru-Guzik, A. (2014) Coherent Exciton Dynamics in Supramolecular Light-Harvesting Nanotubes Revealed by Ultrafast Quantum Process 
Tomography. ACS Nano, 8, 5527-5534. https://doi.org/10.1021/nn406107q

[23] Clark, K.A., Krueger, E.L. and Vanden Bout, D.A. (2014) Direct Measurement of Energy Migration in Supramolecular Carbocyanine Dye Nanotubes. Journal of Physical Chemistry Letters, 5, 2274-2282. https://doi.org/10.1021/jz500634f

[24] Eisele, D.M., Knoester, J., Kirstein, S., Rabe, J.B. and Vanden Bout, D.A. (2009) Uniform Exciton Fluorescence from Individual Molecular Nanotubes Immobilized on Solid Substrates. Nature Nanotechnology, 4, 658-663.

https://doi.org/10.1038/nnano.2009.227

[25] Jaber, M., Robinson, S.W., Missale, C. and Caron, M.G. (1996) Dopamine Receptors and Brain Function. Neuropharmacology, 35, 1503-1519. https://doi.org/10.1016/S0028-3908(96)00100-1

[26] Bowirrat, A. and Oscar-Berman, M. (2005) Relationship between Dopaminergic Neurotransmission, Alcoholism, and Reward Deficiency Syndrome. American Journal of Medical Genetics, 132B, 29-37. https://doi.org/10.1002/ajmg.b.30080

[27] Wiedemann, D.J., Kawagoe, K.T., Kennedy, R.T., Ciolkowski, E.L. and Wightman, R.M. (1991) Strategies for Low Detection Limit Measurements with Cyclic Voltammetry. Analytical Chemistry, 63, 2965-2970.

https://doi.org/10.1021/ac00024a030

[28] Arrigan, D.W., Ghita, M. and Beni, V. (2004) Selective Voltammetric Detection of Dopamine in the Presence of Ascorbate. Chemical Communications, 732-733. https://doi.org/10.1039/b316493d

[29] Park, Y.N., Zhang, X., Rubakhin, S.S. and Sweedler, J.V. (1999) Independent Optimization of Capillary Electrophoresis Separation and Native Fluorescence Detection Conditions for Indolamine and Catecholamine Measurements. Analytical Chemistry, 71, 4997-5002. https://doi.org/10.1021/ac990659r

[30] Thabano, J.R.E., Breadmore, M.C., Hutchinson, J.P., Johns, C. and Haddad, P.R. (2007) Capillary Electrophoresis of Neurotransmitters Using In-Line Solid-Phase Extraction and Preconcentration Using a Methacrylate-Based Weak Cation-Exchange Monolithic Stationary Phase and a $\mathrm{pH}$ Step Gradient. Journal of Chromatography A, 1175, 117-126. https://doi.org/10.1016/j.chroma.2007.09.069

[31] Carrera, V., Sabater, E., Vilanova, E. and Sogorb, M.A. (2007) A Simple and Rapid HPLC-MS Method for the Simultaneous Determination of Epinephrine, Norepinephrine, Dopamine and 5-Hydroxytryptamine: Application to The Secretion of Bovine Chromaffin Cell Cultures. Journal of Chromatography B, 847, 88-94. https://doi.org/10.1016/j.jchromb.2006.09.032

[32] Terech, P. and Talmon, Y. (2002) Aqueous Suspensions of Steroid Nanotubules: Structural and Rheological Characterizations. Langmuir, 18, 7240-7244. https://doi.org/10.1021/la025574r

[33] Terech, P., Jean, B. and Ne, F. (2006) Hexagonally Ordered Ammonium Lithocholate Self-Assembled Nanotubes with Highly Monodisperse Sections. Advanced Materials, 18, 1571-1574. https://doi.org/10.1002/adma.200502358

[34] Pal, A., Basit, H., Sen, S., Aswal, V.K. and Bhattacharya, S.J. (2009) Structure and Properties of Two Component Hydrogels Comprising Lithocholic Acid and Organic Amines. Journal of Materials Chemistry, 19, 4325-4334. https://doi.org/10.1039/b903407b

[35] Zhang, X., Zou, J., Tamhane, K., Kobzeff, F. and Fang, J.Y. (2010) Self-Assembly of pH-Switchable Spiral Tubes: Supramolecular Chemical Springs. Small, 6, 217-220. https://doi.org/10.1002/smll.200901067

[36] Zhang, X., Mathew, M., Gesquiere, A.J. and Fang, J.Y. (2010) Fluorescent Composite Tubes with $\mathrm{pH}$-Controlled Shapes. Journal of Materials Chemistry, 20, 37163721. https://doi.org/10.1039/b927038h 
[37] Wang, H., Xu, W., Song, S., Feng, L., Song, A. and Hao. J. (2014) Unique Temperature-Dependent Supramolecular Self-Assembly: From Hierarchical 1D Nanostructures to Super Hydrogel. Journal of Physical Chemistry B, 118, 4693-4701. https://doi.org/10.1021/jp500113h

[38] Knoester, J. and Agranovich, V.M. (2003) In Electronic Excitations in Organic Based Nanostructures. In: Agranovich, V.M. and Bassani, G.F., Eds., Thin Films and Nanostructures, Elsevier, Amsterdam, Vol. 31.

[39] Sorokin, A.V., Filimonova, I.I., Grynyov, R.S., Guralchuk, G.Y., Yefimova, S.L. and Malyukin, Y.V. (2010) Control of Exciton Migration Efficiency in Disordered J-Aggregates. Journal of physical Chemistry C, 114, 1299-1305. https://doi.org/10.1021/jp906665j

[40] Klegeris, A., Korkina, L.G. and Greenfield, S.A. (1995) Autoxidation of Dopamine: A Comparison of Luminescent and Spectrophotometric Detection in Basic Solutions. Free Radical Biology and Medicine, 8, 215-222. https://doi.org/10.1016/0891-5849(94)00141-6

[41] Medintz, I.L., Stewart, M.H., Trammell, S.A., Susumu, K., Delehanty, J.B., Mei, B.C., Melinger, J.S., Blanco-Canosa, J.B., Dawson, P.E. and Mattoussi, H. (2010) Quantum-Dot/Dopamine Bioconjugates Function as Redox Coupled Assemblies for in vitro and Intracellular pH Sensing. Nature Materials, 9, 676-684. https://doi.org/10.1038/nmat2811

[42] Lee, H., Dellatore, S.M., Miller, W.M. and Messersmith, P.B. (2007) Mussel-Inspired Surface Chemistry for Multifunctional Coatings. Science, 318, 426-430. https://doi.org/10.1126/science.1147241

[43] Bredas, J.L., Silbey, R., Boudreux, D.S. and Chance, R.R. (1983) Chain-Length Dependence of Electronic and Electrochemical Properties of Conjugated Systems: Polyacetylene, Polyphenylene, Polythiophene, and Polypyrrole. Journal of the American Chemical Society, 105, 6555-6559. https://doi.org/10.1021/ja00360a004

[44] Mohamed, M. and Eichborn, S.H. (2010) Measurements and Prediction of Electronic Properties of Discotic Triphenylenes and Phthalocyanines. ECS Transactions, $25,1-10$.

[45] Mohammad-Shiri, H., Ghaemi, M., Riahi, S. and Akbari-Sehat, A. (2011) Computational and Electrochemical Studies on the Redox Reaction of Dopamine in Aqueous Solution. International Journal of Electrochemical Science, 6, 317-336.

[46] Dawson, T.M. and Dawson, V.L. (2003) Molecular Pathways of Neurodegeneration in Parkinson's Disease. Science, 302, 819-822. https://doi.org/10.1126/science.1087753

[47] Zetterstrom, T., Sharp, T., Marsden, C.A. and Ungerstedt, U. (1983) In Vivo Measurement of Dopamine and Its Metabolites by Intracerebral Dialysis: Changes after d-Amphetamine. Journal of Neurochemistry, 41, 1769-1773.

https://doi.org/10.1111/j.1471-4159.1983.tb00893.x 
Submit or recommend next manuscript to SCIRP and we will provide best service for you:

Accepting pre-submission inquiries through Email, Facebook, LinkedIn, Twitter, etc. A wide selection of journals (inclusive of 9 subjects, more than 200 journals)

Providing 24-hour high-quality service

User-friendly online submission system

Fair and swift peer-review system

Efficient typesetting and proofreading procedure

Display of the result of downloads and visits, as well as the number of cited articles Maximum dissemination of your research work

Submit your manuscript at: http://papersubmission.scirp.org/

Or contact msce@scirp.org 